\title{
No difference in the 5-year survival rates between the resin-bonded cantilever bridge and the removable partial denture for the restoration of the shortened dental arch
}

\author{
When restoring shortened lower dental arches, are bilateral cantilever \\ resin-bonded fixed partial dentures more effective than conventional \\ removable partial dentures?
}

\section{Thomason JM, Moynihan PJ, Steen N, Jepson NJ. \\ Time to survival for the restoration of the shortened lower dental arch. J Dent Res 2007; 86:646-650.}

Design This was a randomised controlled trial (RCT) in a dental hospital. Intervention Resin-bonded fixed partial dentures (RBFPD) or removable partial dentures (RPD) were compared. The RBFPD restored 1 occlusal unit, up to but not beyond the second premolar, by means of single pontics cantilevered from single abutments whenever possible. Bilateral lower free-end saddle RPD were constructed on cast metal frameworks that incorporated rests, retainers and a rigid connector. The trial's participants were reviewed 3 months and 1 year after insertion of the new lower prosthesis, and thereafter at yearly intervals. Additional review and maintenance appointments were scheduled as clinically required.

Outcome measure Survival of the prosthesis was assessed at each review according to specified criteria. Survival analysis was performed using a Cox proportional hazard regression ratio.

Results Eleven fixed partial dentures and 15 RPD failed. Debonding was the most common cause of failure in the RBFPD group, with nonuse and loss of teeth most common in the RPD group. Although the RPD group required rather more maintenance visits, the difference in prosthesis survival rates was not statistically significant [hazard ratio (HR), $0.59 ; 95 \%$ confidence intervals $(\mathrm{Cl}), 0.27-1.29]$.

Conclusions The absence of a significant difference in 5-year survival, the reported advantages of fixed partial dentures, and the greater maintenance need for those in the RPD treatment group support the use of RBFPD.
Address for correspondence: JM Thomason, School of Dental Sciences, University of Newcastle upon Tyne, Framlington Place, Newcastle upon Tyne NE2 4BW, UK. E-mail: j.m.thomason@ncl.ac.uk

\section{Commentary}

The term the shortened dental arch was first coined in 1981 by Käyser, ${ }^{1}$ hypothesising (later supported by research evidence) that bilateral bicuspid occlusion (ie, occlusion to the second bicuspid) was an acceptable treatment objective in oral rehabilitation. ${ }^{2}$ The conventional treatment plan for people who present with a complete loss of bilateral or unilateral posterior occlusal stops (neither molar nor bicuspid occlusal stops) has been lower RPD. Unfortunately, this appliance continues to be associated with poor clinical outcomes and patient dissatisfaction..$^{3,4}$

Thomason and colleagues report a generally well-conducted RCT looking at the clinical performance of lower RPD in the management of the shortened dental arch compared with the potentially equally cost-effective and possibly more satisfying alternative, the resinbonded cantilever bridge. This study was carried out as a time-toevent (survival-analysis) RCT rather than just measuring the survival outcomes at a single point in time (only once at the end of the 5-year period). Survival analysis assesses the functionality of each subject's dental appliance at regular followup intervals over the full course of the clinical trial. Such an analysis includes data from participants who withdrew or were lost to followup during the course of the trial period (ie, censored data). Not accounting for the fact that censored data in such an analysis may bias a study's outcome, especially when trials include few participants and/ or the numbers lost to followup are high. ${ }^{5}$ For example, in this study, a quarter (15) of the original 60 patients who were enrolled were lost to followup. By using a KaplanMeier survival analysis and a Cox proportional regression model (typically used in healthcare studies), the investigators were able to justify the inclusion of 54 patients in the final analysis, as opposed to including only 45 patients if the investigators had followed up the patient only once at the end of the 5-year trial.

The statistic generally used in such a time-to-events analysis is the HR. Although similar to the relative risk, censored data are included in its calculation. Therefore, if there are no censored data, then the HR and relative risk would be the same. The HR is this study was determined to be 0.59 . This implies that the probability of failure at anytime was $41 \%$ [(1-0.59) $\times 100]$ lower in the resinbonded cantilever bridge group than in the RPD group. Since the 95\% CI of 0.27-1.29 crosses the point of no difference $(\mathrm{HR}=1)$, the null hypothesis cannot be rejected, and thus it can be statistically inferred that the resin-bonded cantilever bridge performs as poorly, clinically, as the RPD after 5 years. Nevertheless, the authors attempt to make a case in favour of the resin-bonded bridge by reporting that it had fewer treatment needs (specifically, chair side repairs and adjustment) during the course of the 5-year trial than the RPD. 
Some concerns that may threaten the validity of this study are as follows:

- Only 56 of the 60 people included in the study were randomly allocated into the two treatment groups. It is not clear why four subjects were not randomly allocated, and to which groups they were assigned. Although a small part of this study (7\%), if all were assigned to one of the groups this may have favoured - or not - one particular outcome.

- The two clinical operators in this study evaluated their own work. Although the authors acknowledge this potential bias they claim, without elaborating how, that, “... steps [were] taken to avoid this form of error". It would also have been interesting to see the distribution of failures between the two operators. If they were not similar then the evaluated performance of each appliance may have been operator-dependent rather than resulting from the mechanics of the appliances themselves.

- The authors reported a total of 26 failures, comprising 11 in the resin-bonded cantilever bridge group and 15 in the RPD. It is not clear how the authors determined that the, "within-group survival analysis indicates cumulative prosthesis survival of approximately $25 \%$ and $70 \% "$ at 5 years for the RPD and resin-bonded cantilever bridge groups, respectively. Presenting Kaplan-Meier life tables may have helped to clarify this confusion.

Although the a priori sample size calculation is shown, the authors discuss how a post-hoc analysis indicates that repeating this study with 214 participants (107 in each arm of the study) may endow enough power to show a statistical difference. I feel it would be hard to justify funding such a study when the resin-bonded cantilever bridge performs as poorly as the alternative it is trying to replace. Funding might be better-directed towards a qualitative study, quality of life study or cost-benefit analysis dealing with all the currently available treatment options for the shortened dental arch, also including the implants supported by crown or bridge. ${ }^{6}$

\section{Practice point}

A cheap and effective mode of managing the shortened dental arch continues to challenge the clinician.

The resin-bonded cantilever bridge and RPD appear to perform equally poorly, but the former may require less post-delivery care.

\section{Ben Balevi}

Private Practitioner, Vancouver; Affiliated with the Faculty of Medicine, University of British Columbia, Vancouver, Canada

1. Kayser AF. Shortened dental arches and oral function. J Oral Rehabil 1981; 8:457-462.

2. Kanno T, Carlsson GE. A review of the shortened dental arch concept focusing on the work by the Kayser/ Nijmegen group. J Oral Rehabil 2006; 33:850-862.

3. Jepson NJ, Thomason JM, Steele JG. The influence of denture design on patient acceptance of partial dentures. Br Dent J 1995; 178:296-300.

4. Aquilino SA, Shugars DA, Bader JD, White BA. Ten-year survival rates of teeth adjacent to treated and untreated posterior bounded edentulous spaces. J Prosthet Dent 2001; 85:455-460.

5. Chapman D. Modelling survival data in medical research. 2nd Edn. London: Chapman and Hall; 2003.

6. Nassani MZ, Devlin H, McCord JF, Kay EJ. The shortened dental arch - an assessment of patients' dental health state utility values. Int Dent J 2005; 55:307-312.

Evidence-Based Dentistry (2008) 9, 105-106. doi:10.1038/sj.ebd.6400610 